\title{
A computationally efficient technique for the solution of pulp washing models
}

\author{
Satinder Pal Kaur* and Dr. Ajay Kumar Mittal** \\ * Maharaja Ranjit Singh Punjab Technical University, Bathinda, Punjab, India \\ ** Aryabhatta Group of Institutes, Barnala, Punjab, India \\ *Corresponding Author: satinder_pk@yahoo.com
}

$\begin{array}{ll}\text { Submitted } & : 10 / 06 / 2020 \\ \text { Revised } & : 29 / 12 / 2020 \\ \text { Accepted } & : 09 / 01 / 2021\end{array}$

\begin{abstract}
An efficient numerical technique for the solution of the pulp washing model is proposed in this study. Two linear and one nonlinear model are explained with quintic Hermite collocation method (QHCM). In this technique, quintic Hermite polynomials ( $\mathrm{C}^{2}$ continuous) are used as a basis function, and an orthogonal collocation method is applied within each element of the partitioned domain. For accuracy and applicability of the method, a comparison of the numerical results with analytic ones is made. The method is found to be stable using stability analysis and convergence criteria. The effect of Peclet number on exit solute concentration and other parameters is presented in the form of breakthrough curves. The results are derived for a broad range of parameters, and the present method is found to be more efficient and refined for solving the two-point boundary value problems.
\end{abstract}

Keywords: Pulp washing; Collocation; Peclet number; Stability.

\section{INTRODUCTION}

Pulp washing is an important process in the paper industry, which needs to be performed in an ecological and efficient manner. The solute (Na salts and lignin derivatives) inside the pulp matrix is removed by the introduction of wash liquor. The process can be described using material balance equation as follows:

$$
\left\{\begin{array}{l}
\text { Rate of mass } \\
\text { entering } \\
\text { by bulk fluid }
\end{array}\right\}+\left\{\begin{array}{l}
\text { Rate of mass } \\
\text { entering } \\
\text { by dispersion }
\end{array}\right\}=\left\{\begin{array}{l}
\text { Rate of mass } \\
\text { leaving } \\
\text { by bulk fluid }
\end{array}\right\}+\left\{\begin{array}{c}
\text { Rate of mass } \\
\text { by } \\
\text { accumulation }
\end{array}\right\}+\left\{\begin{array}{l}
\text { Rate of mass } \\
\text { accumulated } \\
\text { in solid phase }
\end{array}\right\}
$$

Mathematically, this equation involves different variables and their partial derivatives. These equations along with various linear and nonlinear adsorption isotherms describe the relationship between the concentration of the solute adsorbed on fibers and the solute in the flowing liquor (Kukreja et al.,1995). A detailed review of various models used to describe the pulp washing process has been presented by Pekkanen \& Norden (1985). Besides this, Lapidus \& Amundson (1952), Brenner (1962), Sherman (1964), Grähs (1974), Liu \& Bhatia (2001), and Tervola (2006) have studied these pulp washing models using boundary value problems (BVPs) for different boundary conditions and examined the effect of different parameters on wash efficiency. Brenner (1962) and Kukreja et al. 
(1995) obtained the analytic solution of some models using Laplace transform. A detailed study of these methods revealed that the analytic solution is complex and less suitable for nonlinear problems.

Sun \& Meunier (1991) used the finite difference method (FDM) to solve these models and found that it requires a strict selection of step size for stability. Al-Jabari et al. (1994) and Liu \& Bhatia (2001) solved these models with the Galerkin/Petrov Galerkin method. Some other authors (Villadsen \& Stewart, 1967; Grähs, 1974) used the orthogonal collocation method (OCM) to derive the solution. However, the results obtained using these methods were also not much suitable for a large number of collocation points (Shiraishi, 2001). In addition, the problem of increasing oscillations obtained for the large values of parameters is handled with Orthogonal Collocation on Finite element (OCFE) method. The approximation technique of OCFE is proposed by Carey \& Finlayson (1975) and is used for a large number of interior collocation points. Arora et al. (2005) and Liu \& Jacobsen (2012) used OCFE method and established its accuracy . Further, Ganaie et al. (2013) and Mittal et al. (2013) applied the cubic Hermite collocation method (CHCM) using cubic Hermite polynomials as a trial function, which follows the property of continuity of function and its first-order derivative. They also proved the efficiency and high order of accuracy of this method over OCFE.

In this work, quintic Hermite polynomials (which are $\mathrm{C}^{2}$ continuous) are used as the basis function. Arora et al. (2020) also used this method to solve the Burgers' equation. The number of equations and their mathematical complexity are reduced in this method, because the trial function and their derivatives are continuous at the grid points. To check the accuracy, validity, and applicability of the method, the results are compared with analytic solution obtained by Brenner (1962); Grähs (1974) and numerical solution of Mittal et al. (2013). Thereafter, the technique is extended to the nonlinear problem and to examine the effect of fundamental parameters on exit concentration profiles of the adsorbed solute. The procedure used for the selection of collocation points, stability analysis, and convergence criteria are discussed in the next sections.

\section{PROBLEM FORMULATION}

This section describes the model equations to be solved using the technique of QHCM.

\section{Linear Model 1}

Consider the dimensionless form of the linear model representing diffusion dispersion problem with Dirichlet's boundary conditions as

$$
\frac{\partial C}{\partial T}=\frac{1}{P e} \frac{\partial^{2} C}{\partial \xi^{2}}-\frac{\partial C}{\partial \xi} \quad \text { in } \quad \Omega \in(0,1)
$$

$$
C=0 \quad \text { at } \xi=0
$$

Boundary condition: $\frac{\partial C}{\partial \xi}=0$

$$
\left.\begin{array}{l}
\text { at } \xi=0 \\
\text { at } \xi=1
\end{array}\right\} \text { for all } T \geq 0
$$

Initial condition: $\quad C(\xi, 0)=1$

Grähs (1974) solved the above problem with OCM and found the analytic solution to this problem. However, Arora et al. (2005) efficiently solved the model with OCFE method and achieved better results than OCM. Further, 
Mittal et al. (2013) obtained the numerical solution of this problem with CHCM and found the technique of CHCM more suitable than OCFE.

\section{Linear Model 2}

Consider the linear model 1 with mixed boundary conditions defined as

$$
\left.\begin{array}{ll}
P e C=\frac{\partial C}{\partial \xi} & \text { at } \xi=0 \\
\frac{\partial C}{\partial \xi}=0 & \text { at } \xi=1
\end{array}\right\} \text { for all } T \geq 0
$$

Brenner (1962) solved this problem by Laplace transform method. Further, Arora et al. (2005) solved the model with OCFE. Both studies found satisfactory results.

\section{Nonlinear model}

The unsteady state partial differential equation describing the phenomenon of longitudinal mixing is given as follows:

$$
D_{L} \frac{\partial^{2} c}{\partial z^{2}}=U \frac{\partial c}{\partial z}+\frac{\partial c}{\partial t}+C_{F}\left(\frac{1-\varepsilon}{\varepsilon}\right) \frac{\partial n}{\partial t}
$$

Nonlinear Langmuir adsorption isotherm is $n=\frac{A_{0} c}{1+B_{0} c}$

where $A_{0}$ and $B_{0}$ are Langmuir constants. Boundary conditions and initial conditions assumed are the same as those in linear model 2. The dimensionless form of the model is

$$
\frac{1}{P e} \frac{\partial^{2} C}{\partial \xi^{2}}=\frac{\partial C}{\partial \xi}+\frac{\partial C}{\partial T}+\frac{\mu C_{F} A_{0}}{\left[1+B_{0}\left\{C\left(C_{0}-C_{s}\right)+C_{s}\right\}\right]^{2}} \frac{\partial C}{\partial T}
$$

Earlier, these model equations with linear isotherm were solved by Kukreja \& Ray (2009) using the Laplace Transform method. Further, Arora (2005) solved the nonlinear problem using OCFE, and Mittal et al. (2013) obtained the solution of the above problem using CHCM. All these studies achieved notable results.

\section{QUINTIC HERMITE COLLOCATION METHOD (QHCM)}

In the present study, quintic Hermite polynomials are taken as basis function in OCM to solve the above problems. The subsidiary continuity condition of first and second derivatives at the boundary of each element is not required in these polynomials; therefore, the number of equations is reduced considerably (Dyksen \& Lynch, 2000). The domain $0 \leq \xi \leq 1$ is divided into a finite number of subparts called elements by inserting $\xi_{1}, \xi_{2}, \ldots, \xi_{N+1}$ points such that $\xi_{1}=0$ and $\xi_{N+1}=1$ with $h_{k}=\xi_{k+1}-\xi_{k}$. A new variable $u=\left(\xi-\xi_{k}\right) / h_{k}$ is 
introduced such that $u$ varies from 0 to 1 when $\xi$ varies from $\xi_{k}$ to $\xi_{k+1}$. Thereafter, OCM is applied within each element for the 4 collocation points with $\mathrm{N}$ intervals.

The approximate solution $c(u, t)$ and its partial derivatives at $r^{\text {th }}$ collocation point in the $k^{\text {th }}$ element are given by

$c_{k r}(u, t)=\sum_{q=1}^{6} a_{q+3(k-1)}^{r}(t) H_{q}^{k}\left(u_{r}\right) ; k=1,2, \ldots, N ; r=2,3,4,5$.

$\frac{\partial c_{k r}}{\partial u}=\frac{1}{h_{k}} \sum_{q=1}^{6} a_{q+3(k-1)}^{r} \frac{d H_{q}^{k}}{d u}$

$\frac{\partial^{2} c_{k r}}{\partial u^{2}}=\frac{1}{h_{k}^{2}} \sum_{q=1}^{6} a_{q+3(k-1)}^{r} \frac{d^{2} H_{q}^{k}}{d u^{2}}$

$\frac{\partial c_{k r}}{\partial t}=\sum_{q=1}^{6} \frac{d a_{q+3(k-1)}^{r}}{d t} H_{q}^{k}$

where the detailed description of standard quintic Hermite basis functions $H_{q}^{k}$ 's is available with Kaur et al. (2018).

\section{Selection of Collocation Points}

The selection of collocation points plays a substantial role in the collocation techniques (Villadsen \& Stewart, 1967). These points are taken as the roots of orthogonal polynomials. In QHCM, zeros of $4^{\text {th }}$ order shifted Legendre polynomial are used as collocation points. These polynomials are a special case of orthogonal polynomial such as Jacobi polynomials and give accurate results (Andrews, 1984).

\section{Discretized form of Model}

Discretized form of the linear model is as follows:

$$
\sum_{q=1}^{6} \frac{d a_{q+3(k-1)}^{r}}{d t} H_{q}^{k}\left(u_{r}\right)=\frac{1}{P e h_{k}^{2}} \sum_{q=1}^{6} a_{q+3(k-1)}^{r} H_{q}^{k^{\prime \prime}}\left(u_{r}\right)-\frac{1}{h_{k}} \sum_{q=1}^{6} a_{q+3(k-1)}^{r} H_{q}^{k^{\prime}}\left(u_{r}\right)
$$

where $r=2,3,4,5$ (interior collocation points) and $k=1,2, \ldots, N$ (number of elements).

Discretized form of nonlinear model is as follows: 


$$
\begin{aligned}
\sum_{q=1}^{6} \frac{d a_{q+3(k-1)}^{r}}{d t} H_{q}^{k}\left(u_{r}\right)= & {\left[\frac{\left[1+B_{0}\left\{\left(C_{0}-C_{s}\right) \sum_{q=1}^{6} a_{q+3(k-1)}^{r} H_{q}^{k}\left(u_{r}\right)+C_{s}\right\}\right]^{2}}{\mu C_{F} A_{0}+\left[1+B_{0}\left\{\left(C_{0}-C_{s}\right) \sum_{q=1}^{6} a_{q+3(k-1)}^{r} H_{q}^{k}\left(u_{r}\right)+C_{s}\right\}\right]^{2}}\right] } \\
& \times\left(\frac{1}{P e h_{k}^{2}} \sum_{q=1}^{6} a_{q+3(k-1)}^{r} H_{q}^{k^{\prime \prime}}\left(u_{r}\right)-\frac{1}{h_{k}} \sum_{q=1}^{6} a_{q+3(k-1)}^{r} H_{q}^{k^{\prime}}\left(u_{r}\right)\right)
\end{aligned}
$$

where $r=2,3,4,5$ (interior collocation points) and $k=1,2, \ldots, N$ (number of elements).

By the technique of QHCM, the above system of partial differential equations is reduced into a system of ordinary differential equations and is solved using MATLAB's ode 15s.

\section{Error and Convergence Criteria}

Arora \& Kaur (2020) tested the stability of the method using Euclidean norms and supremum norms given as follows:

$$
\|L\|_{2}=\sqrt{h \sum_{j=1}^{N} E^{2}} \text { and }\|L\|_{\infty}=\max _{j} E
$$

where the point-wise error is defined by $E$ such that $E=\left|c_{j}^{\text {exact }}-\left(c_{N}\right)_{j}\right|$ for $c_{j}^{\text {exact }}$ is the exact solution and $\left(c_{N}\right)_{j}$ is the numerical solution. According to Edoh et al. (2000), it is not an easy task to calculate the residual for nonlinear BVPs. However, according to Arora et al. (2005), the stability analysis for these models can be checked on the basis of numerical results obtained using $\|L\|_{2} \leq 1$. Further, Onah (2002) explained that the time-dependent function converges to a steady-state solution when $t \rightarrow \infty$.

\section{RESULTS AND DISCUSSION}

In this section, the results of this study are described in detail. The results of linear and nonlinear problems are obtained using the technique of QHCM and CHCM. Thereafter, the comparison between the two methods is given for different parameters. The stability, convergence, and efficiency of the method are discussed and compared with the previous techniques in the next paragraphs.

\section{Comparison Between Analytic and Numerical Results}

The numerical results obtained by dividing the domain into 30 elements from linear model 1 using QHCM are compared with the analytic results given by Grähs (1974) for Peclet number $(\mathrm{Pe})=1,10$, and 40 and presented in Figure 1. It is seen that numerical results match best with analytic ones. Also, with the increase in time, the solution profile converges to steady-state condition, which validates the convergence criteria. 


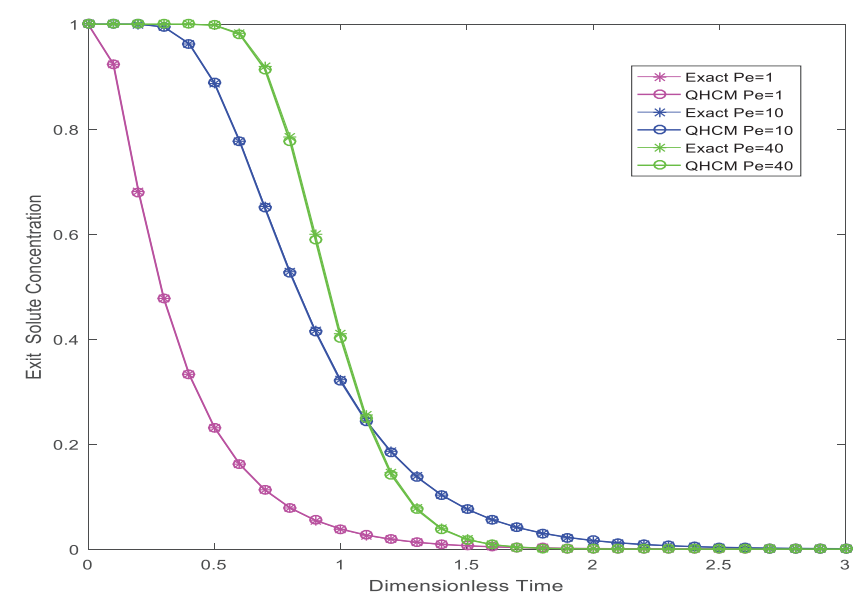

Figure 1. Comparison of exact solution and QHCM.

\section{Comparison of Relative Error Between QHCM and CHCM}

The accuracy of the results can be checked by calculating the relative error. The analytic solution of linear model 1 is obtained by Grähs (1974). The numerical solution, attained using QHCM, is compared with analytic one in terms of relative error. The results of relative error using QHCM and CHCM are graphically shown in Figure 2(a). It is observed that the relative error decreases with the increase in the number of elements. It can be seen that for $\mathrm{Pe}=1$, the relative error in case of $\mathrm{CHCM}$ with 10 elements fluctuates with a maximum magnitude of $1.4 \times 10^{-1}$ (approximately).

Brenner (1962) obtained the analytic solution for linear model 2 with the method of Laplace transform. The relative error between the exact and numerical results is plotted in Figure 2(b). Further, it is observed that the relative error is nearer to zero in QHCM, whereas, in CHCM, its magnitude is nearly $7 \times 10^{-8}$. Hence, the supremacy of the method over previous work is confirmed with error analysis.

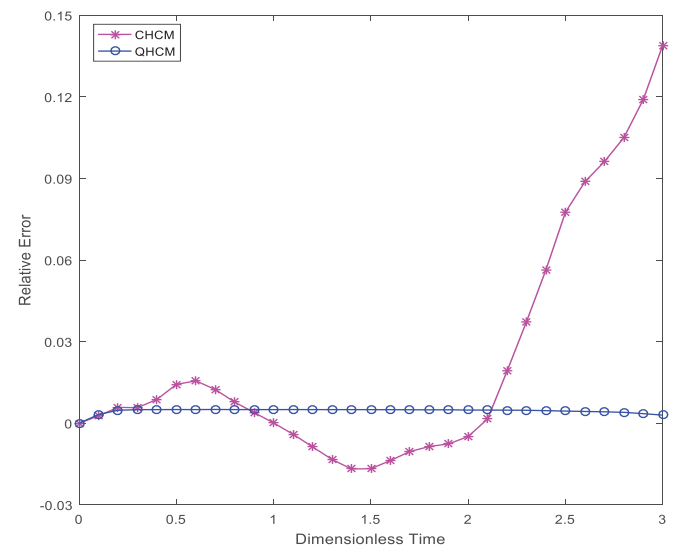

Fig 2(a)

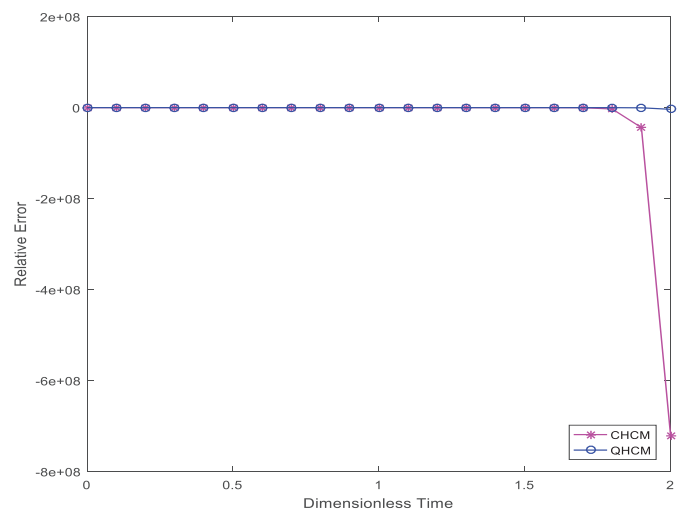

Fig 2(b)

Figure 2(a). Comparison of relative error of $\mathrm{CHCM}$ and $\mathrm{QHCM}$ for $\mathrm{Pe}=1$ and $\mathrm{M}=10$.

2(b). Comparison of relative error of $\mathrm{CHCM}$ and $\mathrm{QHCM}$ for $\mathrm{Pe}=40$ and $\mathrm{M}=10$. 


\section{Stability Analysis Using Norms and Comparison with CHCM}

The stability analysis for QHCM using $\|L\|_{2}$ (Euclidean) and $\|L\|_{2}$ (maximum) norms for both linear models is presented below. The maximum absolute error of QHCM is obtained by using the exact solution given by Grähs (1974) for linear model 1 and by Brenner (1962) for linear model 2 for a different range of Peclet number (Pe). Thereafter, the comparison is drawn with the technique CHCM used by Mittal et al. (2013) in Table 1. It is observed that the error derived for both the norms using CHCM is greater than that by using QHCM in all the cases for both models. Also, both norms lie between 0 and 1. Hence, QHCM is found to be more stable than CHCM. Keeping the above aspects in view, it can be concluded that QHCM is a better technique to solve the BVPs.

Table 1. Comparison of $\|L\|_{2}$ and $\|L\|_{2}$ norms using CHCM and QHCM for $N=40$.

\begin{tabular}{|c|c|c|c|c|c|c|c|c|}
\hline \multirow{4}{*}{} & \multicolumn{4}{|c|}{ Linear model 1 } & \multicolumn{4}{c|}{ Linear model 2 } \\
\cline { 2 - 10 } & \multicolumn{2}{|c|}{$\|L\|_{\infty}$} & \multicolumn{2}{|c|}{$\|L\|_{2}$} & \multicolumn{2}{|c|}{$\|L\|_{\infty}$} \\
\cline { 2 - 9 } & $\mathrm{CHCM}$ & QHCM & CHCM & QHCM & CHCM & QHCM & CHCM & QHCM \\
\hline $\mathrm{Pe}=1$ & $2.514 \mathrm{E}-03$ & $5.804 \mathrm{E}-04$ & $6.722 \mathrm{E}-04$ & $2.856 \mathrm{E}-04$ & $1.643 \mathrm{E}-01$ & $1.642 \mathrm{E}-02$ & $5.782 \mathrm{E}-02$ & $4.782 \mathrm{E}-02$ \\
\hline $\mathrm{Pe}=10$ & $2.640 \mathrm{E}-03$ & $2.200 \mathrm{E}-03$ & $8.872 \mathrm{E}-04$ & $7.117 \mathrm{E}-04$ & $2.053 \mathrm{E}-01$ & $2.072 \mathrm{E}-02$ & $6.735 \mathrm{E}-02$ & $5.73 \mathrm{E}-02$ \\
\hline $\mathrm{Pe}=40$ & $2.682 \mathrm{E}-01$ & $7.552 \mathrm{E}-03$ & $7.974 \mathrm{E}-02$ & $2.175 \mathrm{E}-03$ & $1.912 \mathrm{E}-01$ & $1.684 \mathrm{E}-02$ & $5.157 \mathrm{E}-02$ & $4.892 \mathrm{E}-02$ \\
\hline
\end{tabular}

\section{Effect of Peclet Number (Pe) on Exit Solute Concentration}

The mathematical models explained above are simulated using the data of Grähs (1974). The results are derived for different values of Pe on exit solute concentration profile for the nonlinear model using QHCM. Figure 3 depicted that, for small value of $\mathrm{Pe}$, a longer time is taken by the solution profile to converge and to reach steady-state condition. In this state, the bed behaves like a perfect mixing chamber and black liquor instantly starts coming out. However, with an increase in Pe, dispersion coefficient is decreasing, and the black liquor takes less time in recovery. Although removing all the impurities from the packed bed is a difficult task, however, for Pe $>30$, liquor can be washed out in lesser time. Al- Jabari et al. (1994) supported that efficient washing is attained for Pe between 30 and 50. The numerical results obtained by the QHCM approaches a steady-state condition when time increases.

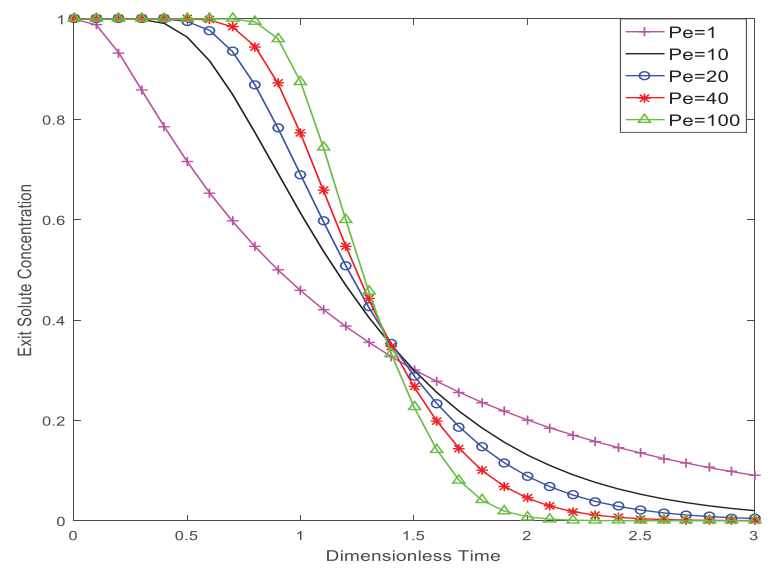

Figure 3. Comparison of exit solute concentration for the number of elements 32 . 


\section{Rate of Convergence}

The rate of convergence of the method for the nonlinear model is calculated using the work of Farrell and Hegarty (1991). The comparison of the rate of convergence for different values of Pe is presented in Table 2. In all the cases, the rate of convergence is found to be nearer to 1. Also, it can be observed that QHCM gives a better rate of convergence than CHCM. It proves the efficiency of the method.

Table 2. Comparison of rate of convergence of CHCM and QHCM.

\begin{tabular}{|c|c|c|c|c|c|c|}
\hline & \multicolumn{2}{|c|}{ Pe=40 } & \multicolumn{2}{c|}{ Pe=100 } & \multicolumn{2}{c|}{ Pe=300 } \\
\hline N (No. of elements) & QHCM & CHCM & QHCM & CHCM & QHCM & CHCM \\
\hline 2 & 1.185371 & 1.18383 & 1.01612 & 0.962791 & 0.898063 & 0.721321 \\
\hline 4 & 1.121715 & 1.111741 & 1.337504 & 1.330525 & 0.976559 & 0.971066 \\
\hline 8 & 1.003269 & 0.807817 & 1.114903 & 1.076677 & 1.466885 & 1.463702 \\
\hline 16 & 0.989565 & 0.846704 & 0.985348 & 0.734532 & 1.322787 & 1.302781 \\
\hline
\end{tabular}

\section{Effect of Displacement Ratio (DR)}

The displacement ratio is a common performance parameter used in Industry. It signifies the actual reduction in black liquor solids relative to maximum possible reduction. The effect of DR for different values of Pe in the form of the breakthrough curve is shown in Figure 4. It is observed that, for a large value of the Pe, more quantity of black liquor solid is removed, because dispersion is small in the case when the Pe is large. Due to this, less back mixing occurs, and the impurities adsorbed on fiber are removed in large quantity in a short period. When the value of DR is 1 , the $100 \%$ solids are assumed to be removed from the packed bed, although it is not possible in real sense. Thus, for the high value of Pe, maximum reduction of dissolved solids is possible and better washing can be achieved.

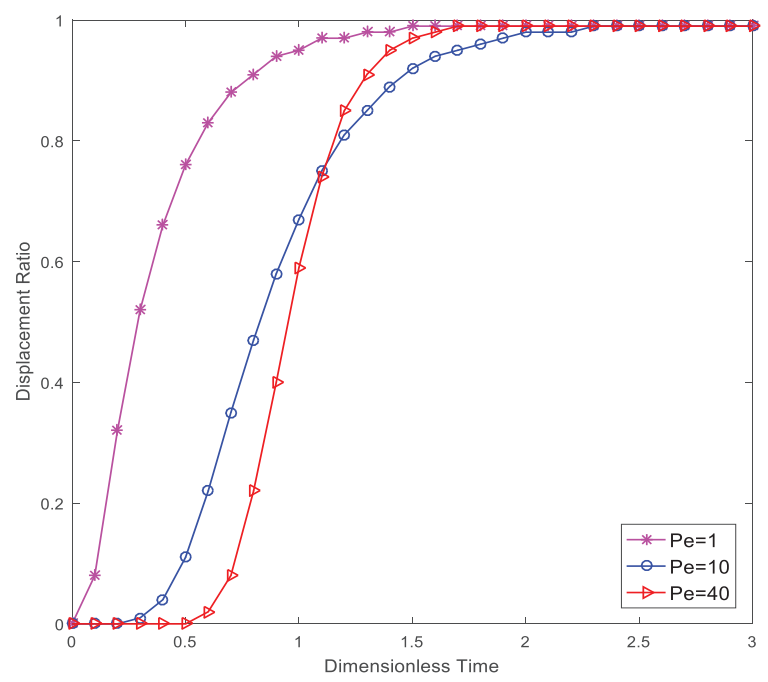

Figure 4. Effect of Peclet number on Displacement ratio. 


\section{Effect of Bed Efficiency}

During the washing operation, bed efficiency gives the net change in the quantity of black liquor solids Efficiency is determined on the basis of quantity of black liquor solids removed. It is observed from Figure 5 that efficiency is increased with the increase in Pe. Hence, the washing operation is influenced by Pe. Also, the efficiency is increased with a high Pe in comparison to a small Pe.

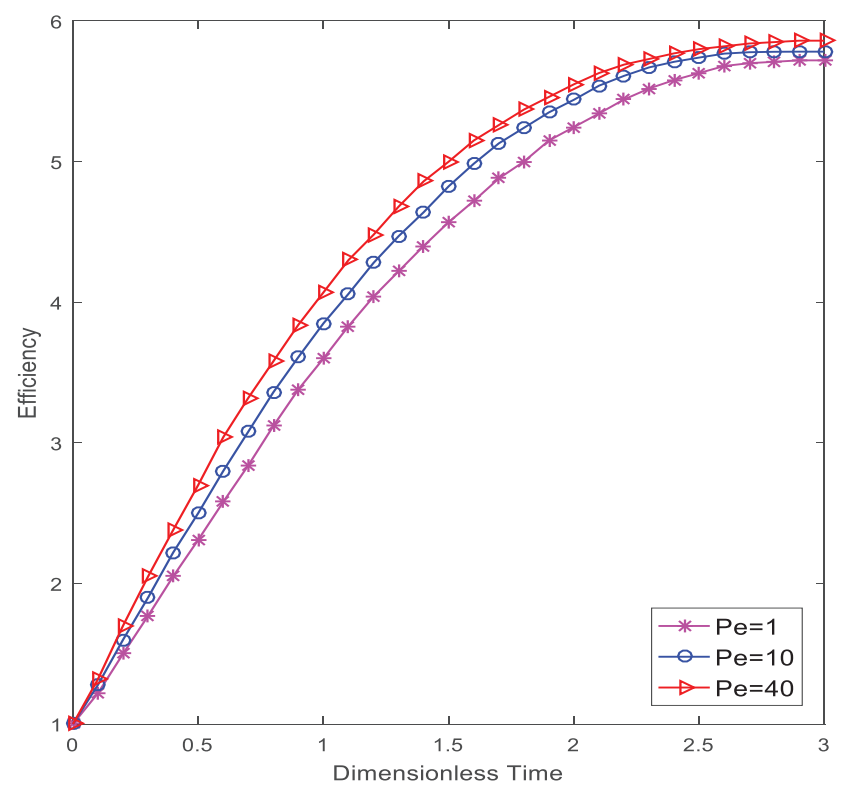

Figure 5. Effect of Peclet number on bed efficiency.

\section{CONCLUSION}

In the present work, the QHCM is proposed to find the numerical solution of linear and nonlinear models of pulp washing. The results attained for the linear models using QHCM are found to keep pace with the analytic solution given by earlier studies. The relative error as compared to previous techniques ( $\mathrm{CHCM})$ is noticed minimum. Euclidean and supremum norms are used to establish the stability of the method. Afterwards, this technique is employed to solve the nonlinear model related to the washing of the pulp fiber bed. The Pe is found to have a notable effect on the concentration of solute at the exit level. The rate of convergence is also obtained that is nearer to 1 . The industrial parameters such as displacement ratio and bed efficiency are also discussed. It is observed that the axial dispersion coefficient considerably affects the concentration profile. Keeping in view all of the mentioned aspects, the QHCM is an effective technique and gives more accurate results. Hence, this method can be efficiently used for solving linear and nonlinear BVPs. 


\section{REFERENCES}

Al-Jabari, M., Van Heiningen, A. R. P., \& Van De Ven, T. G. M. 1994. Modelling the flow and the deposition of fillers in packed beds of pulp fibres. Journal of Pulp and Paper Science, 20(9): J249-J253.

Andrews, L. C. 1984. Special functions for Engineers and Applied Mathematicians. Macmillan Pub Co., New York.

Arora, S., Dhaliwal, S. S., \& Kukreja, V. K. 2005. Solution of two-point boundary value problems using orthogonal collocation on finite elements. Applied Mathematics and Computation, 171(1): 358-370.

Arora, S., Kaur, I., \& Tilahun, W. 2020. An exploration of quintic Hermite splines to solve Burgers' equation. Arabian Journal of Mathematics, 9(1): 19-36.

Brenner, H. 1962. The diffusion model of longitudinal mixing in beds of finite length. Numerical values. Chemical Engineering Science, 17(4): 229-243.

Carey, G. F., \& Finlayson, B. A. 1975. Orthogonal collocation on finite elements. Chemical Engineering Science, 30(5-6): 587-596.

Dyksen, W. R., \& Lynch, R. E. 2000. A new decoupling technique for the Hermite cubic collocation equations arising from boundary value problems. Mathematics and Computers in Simulation, 54(4-5): 359-372.

Edoh, K. D., Russell, R. D., \& Sun, W. 2000. Computation of invariant tori by orthogonal collocation. Applied Numerical Mathematics, 32(3): 273-289.

Farrell, P. A., \& Hegarty, A. 1991. On the determination of the order of uniform convergence. In Proceedings of IMACS World Congress Dublin, Ireland, 501-502.

Ganaie, I. A., Gupta, B., Parumasur, N., Singh, P., \& Kukreja, V. K. 2013. Asymptotic convergence of cubic Hermite collocation method for parabolic partial differential equation. Applied Mathematics and Computation, 220: 560-567

Grähs, L. E. 1974. Washing of cellulose fibres: analysis of the displacement washing operation. PhD Thesis, Chalmers University of Technology, Goteborg, Sweden.

Kaur, S. P., Mittal, A. K., Kukreja, V. K., Parumasur, N., \& Singh, P. 2018. An efficient technique for solution of linear and nonlinear diffusion-dispersion models. AIP Conference Proceedings, 1975 (1): 030031- 1-10.

Kukreja, V. K., Ray, A. K., Singh, V. P., \& Rao, N. J. 1995. A Mathematical model for pulp washing in different zones of a rotary vacuum filter, Journal of Indian Institute of Chemical Engineers, 37: 113-124.

Kukreja, V. K., \& Ray, A. K. 2009. Mathematical modelling of a rotary vacuum washer used for pulp washing: a case study of a lab scale washer. Cellulose Chemistry \& Technology, 43(1): 25-36.

Lapidus, L., \& Amundson, N. R. 1952. Mathematics of adsorption in beds. VI. The effect of longitudinal diffusion in ion exchange and chromatographic columns. The Journal of Physical Chemistry, 56(8): 984-988.

Liu, F., \& Bhatia, S. K. 2001. Application of Petrov-Galerkin methods to transient boundary value problems in chemical engineering: adsorption with steep gradients in bidisperse solids. Chemical Engineering Science, 56(12): 3727-3735.

Liu, Y., \& Jacobsen, E. W. 2004. On the use of reduced order models in bifurcation analysis of distributed parameter systems. Computers \& Chemical Engineering, 28(1-2): 161-169.

Mittal, A. K., Ganaie, I. A., Kukreja, V. K., Parumasur, N., \& Singh, P. 2013. Solution of diffusion-dispersion models using a computationally efficient technique of orthogonal collocation on finite elements with cubic Hermite as basis. Computers \& Chemical Engineering, 58: 203-210.

Onah, S. E. 2002. Asymptotic behaviour of the Galerkin and the finite element collocation methods for a parabolic equation. Applied Mathematics and Computation, 127(2-3): 207-213 
Pekkanen, M., \& Norden, H. V. 1985. Review of pulp washing models. Paperi ja puu, 67(11): 689-696.

Sherman, W. R. 1964. The movement of a soluble material during the washing of a bed of packed solids. AIChE Journal, 10(6): 855-860.

Shiraishi, F. 2001. Highly accurate solution of the axial dispersion model expressed in S-system canonical form by Taylor series method. Chemical Engineering Journal, 83(3): 175-183.

Sun, L. M., \& Meunier, F. 1991. An improved finite difference method for fixed bed multicomponent sorption. AIChE journal, 37(2): 244-254.

Tervola, P. 2006. Fourier series solution for multistage counter current cake washing and segregated wash effluent circulation. Chemical Engineering Science, 61(10): 3268-3277.

Villadsen, J. V., \& Stewart, W. E. 1967. Solution of boundary-value problems by orthogonal collocation. Chemical Engineering Science, 22(11): 1483-1501. 